\title{
Numerical study of flow across an ellipse and a circle placed in a uniform stream of infinite extent
}

\author{
Adnan Anwar ${ }^{1}$, Mudassar Razzaq $^{2}$, and Liudmila Rivkind ${ }^{2}$ \\ ${ }^{1}$ Lahore University of Management Sciences \\ ${ }^{2}$ Institute of Applied Mathematics, TU Dortmund, Germany
}

December 25, 2020

\begin{abstract}
As an example of an aerodynamics prototypical study, we examined a two-dimensional low Reynolds number flow over obstacles immersed in a stream of infinite extent. The Navier Stokes equation is being discretized by non conforming finite element method approach. The resulting discretized nonlinear algebraic system is being solved by using the fixpoint method and the Newton method and multigrid method for the linear sub-problem employed. The magnitude of the uniform upstream velocity under the study of the problem for Reynolds number in the range $1<\operatorname{Re}<100$ and the angle of attack of the upstream velocity at $\alpha=-5 ; 0 ; 5$ degrees performed. Analysis of the resulting drag and lift forces acting on obstacles with respect to the angle of attack of the upstream velocity and the Reynolds number is made. Moreover, the influence of one obstacle on the resulting drag and lift coefficients of other obstacles determined. The results are being presented in a graphical and vector form.
\end{abstract}

\section{Hosted file}

MudassarAdnanRivkin.pdf available at https://authorea.com/users/385175/articles/500645numerical-study-of-flow-across-an-ellipse-and-a-circle-placed-in-a-uniform-stream-ofinfinite-extent 
figures/f1/f1-eps-converted-to.pdf 
figures/f2/f2-eps-converted-to.pdf 
figures/f3/f3-eps-converted-to.pdf 
figures/f4/f4-eps-converted-to.pdf 
figures/f5/f5-eps-converted-to.pdf 
figures/f6/f6-eps-converted-to.pdf

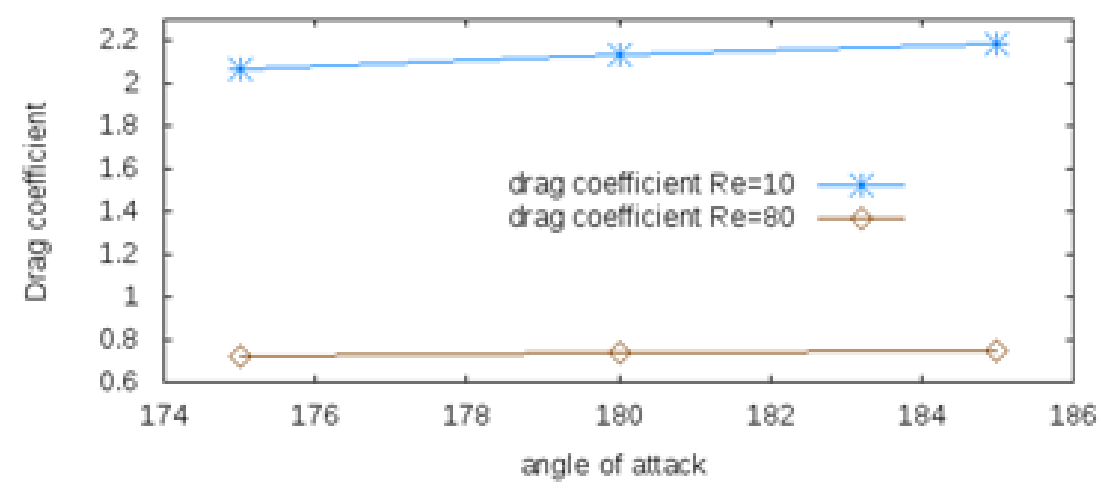



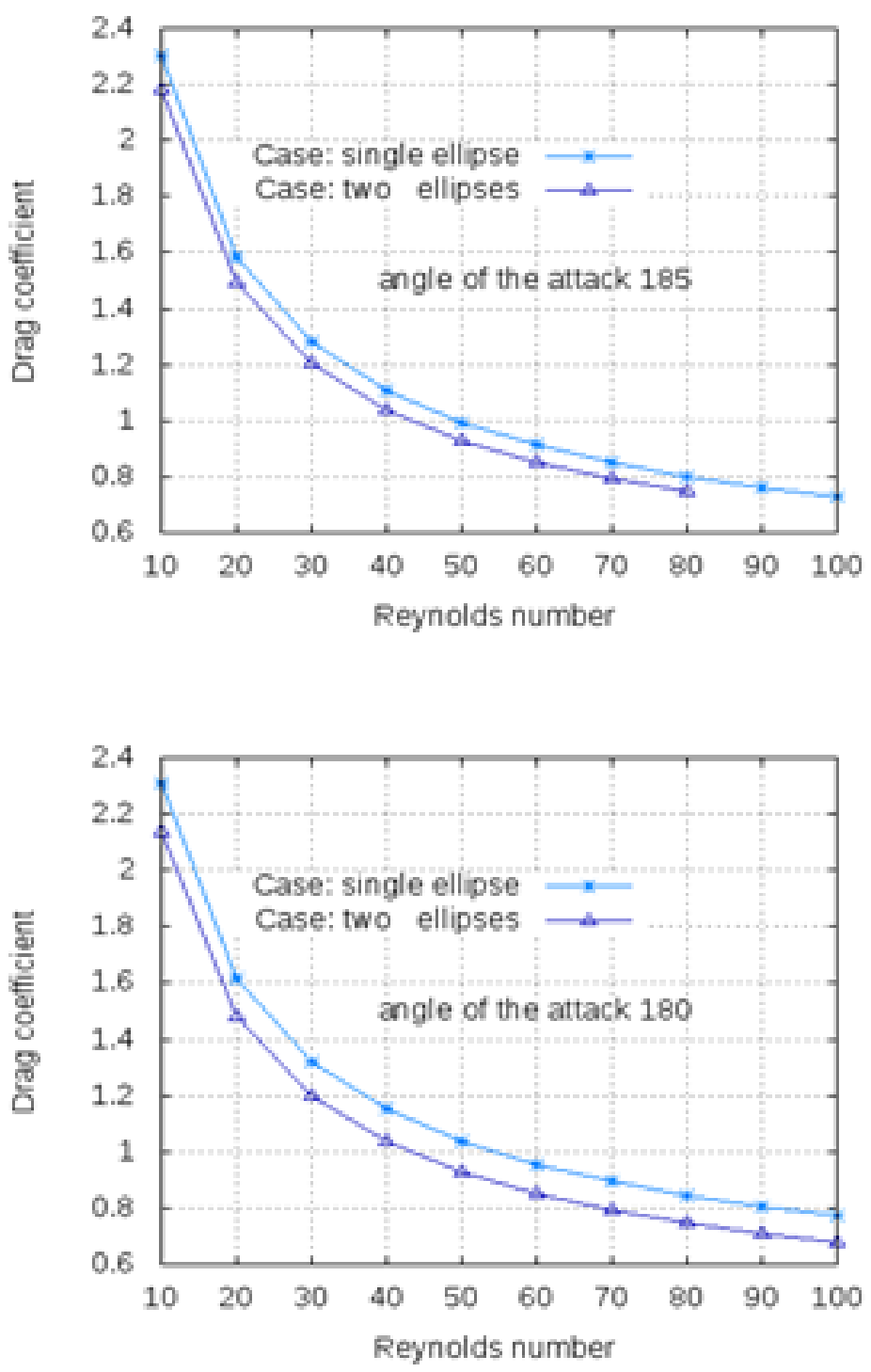

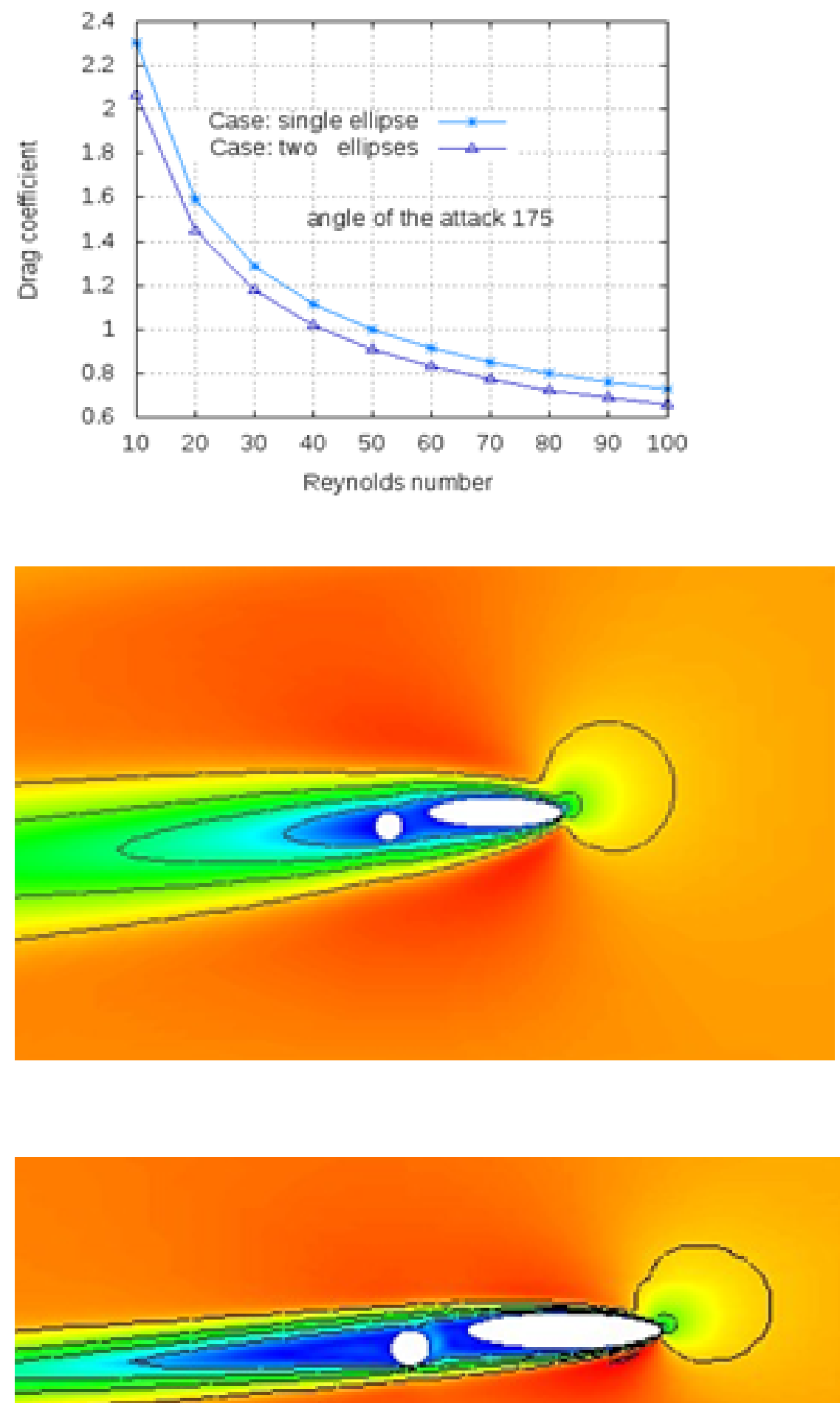

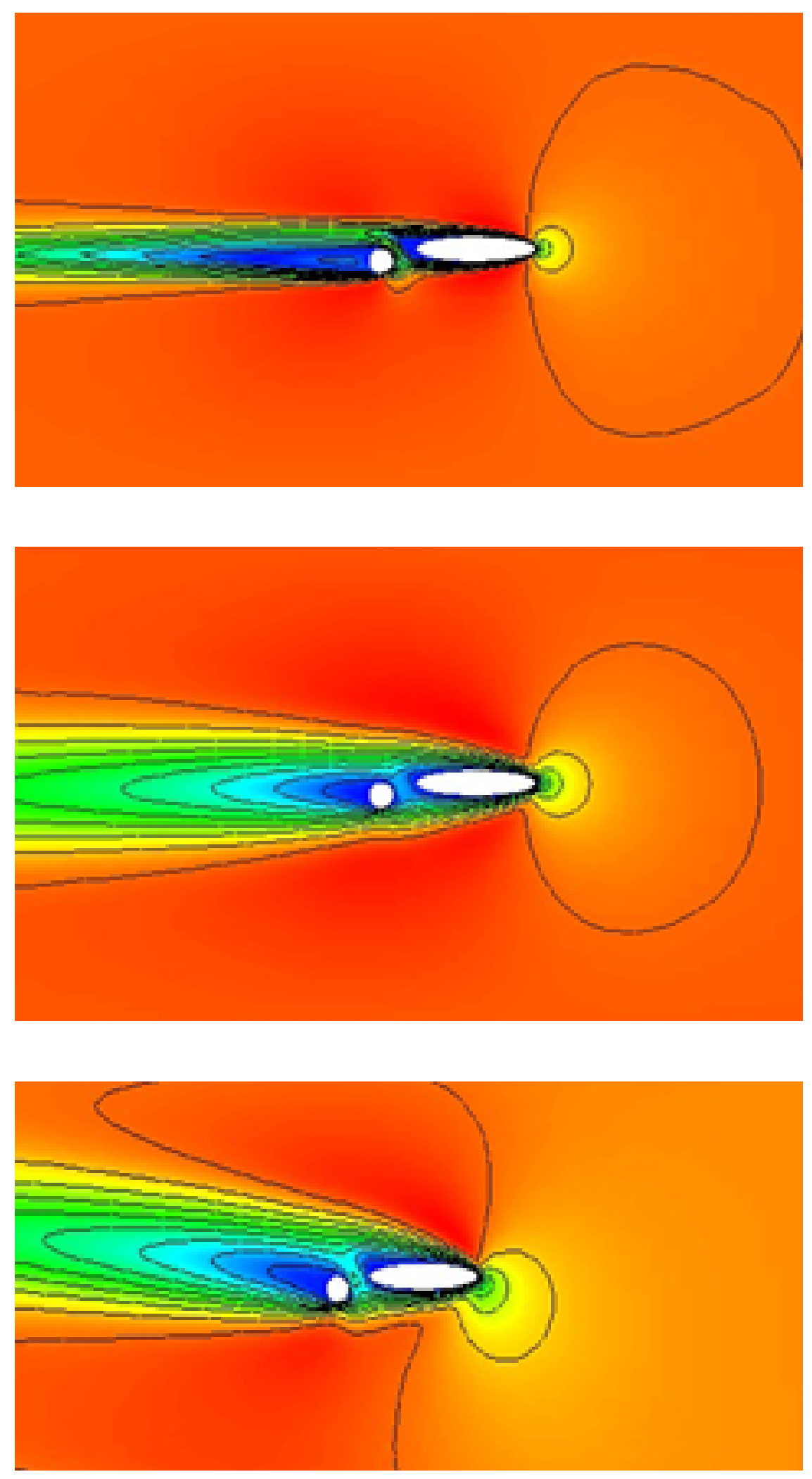

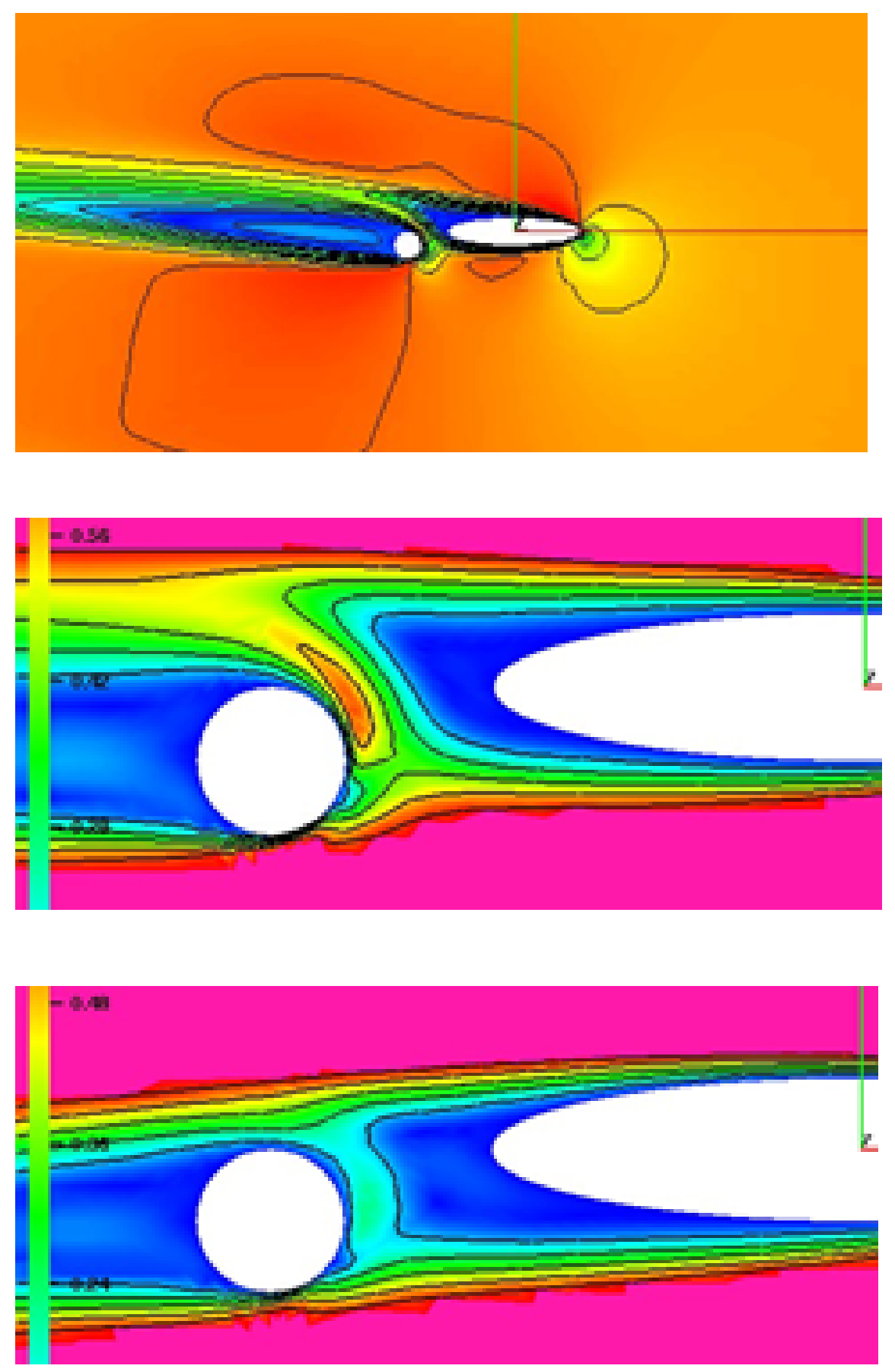


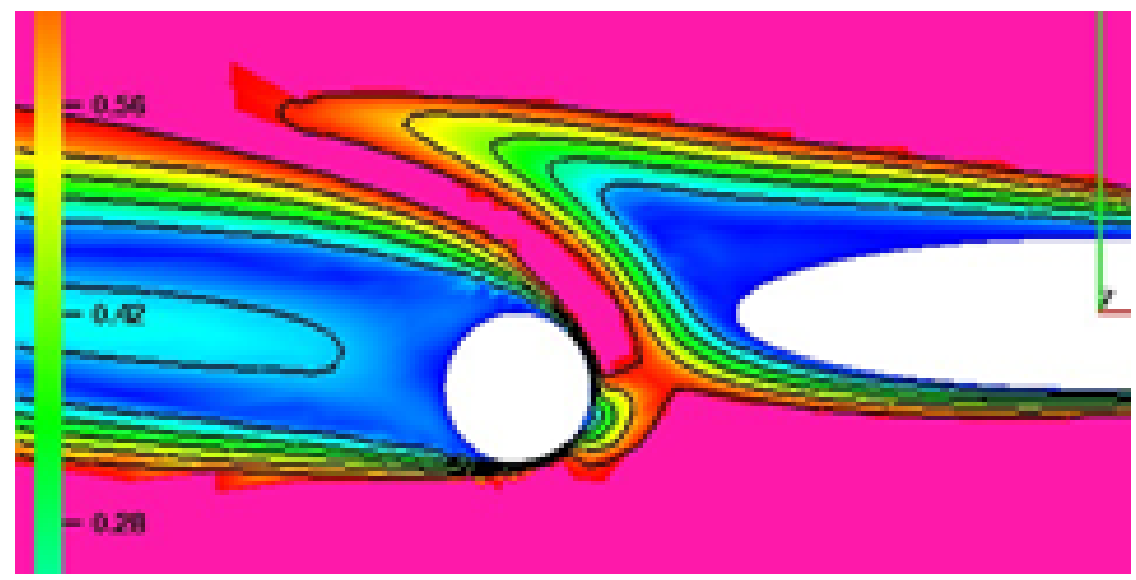

